\title{
Clinical Encounter
}

National Cancer Institute

\section{Source}

National Cancer Institute. Clinical Encounter. NCI Thesaurus. Code C142427.

Any physical or virtual contact between a patient (or trial subject) and healthcare provider at which an assessment or activity takes place. 